\title{
Seismo-Ionospheric Precursors of the 26 December 2006 M 7.0 Pingtung Earthquake Doublet
}

\author{
Jann-Yenq Liu ${ }^{1,2, *}$, Sen-Wen Chen ${ }^{1}$, Yao-Chun Chen ${ }^{1}$, Horng-Yuan Yen ${ }^{3}$, Chung-Pai Chang ${ }^{2,3}$, \\ Wen-Yen Chang ${ }^{4}$, Lung-Chih Tsai ${ }^{1,2}$, Chia-Hung Chen ${ }^{1}$, and Wen-Hsi Yang ${ }^{1}$ \\ ${ }^{1}$ Institute of Space Science, National Central University, Chung-Li, Taiwan, ROC \\ ${ }^{2}$ Center for Space and Remote Sensing Research, National Central University, Chung-Li, Taiwan, ROC \\ ${ }^{3}$ Department of Earth Science and Institute of Geophysics, National Central University, Chung-Li, Taiwan, ROC \\ ${ }^{4}$ Department of Natural Science, National Science Council, Taipei, Taiwan, ROC
}

Received 14 January 2008, accepted 26 September 2008

\begin{abstract}
A network of ground-based GPS receivers, an ionosonde, and a Doppler sounding system have been employed to monitor the total electron content (TEC), electron density, and vertical motion of the ionosphere above Taiwan. Two M 7.0 offshore earthquakes occurred near Pingtung, the most southern county of Taiwan, on 26 December 2006. Analysis has revealed that the ionospheric TEC and F2-pear electron density $(\mathrm{NmF} 2)$ surrounding the epicenters of the events decreased abnormally in the afternoon of the fourth day before the earthquakes, and quasi 3-minute fluctuations of pronounced vertical motion in the ionosphere also appeared over the last two days prior to the earthquakes. This is for the first time the pre-earthquake fluctuation signatures in the ionosphere being reported.
\end{abstract}

Key works: Seismo-ionospheric precursor, Ionosphere, Total electron content, NmF2, Doppler sounding system

Citation: Liu, J. Y., S. W. Chen, Y. C. Chen, H. Y. Yen, C. P. Chang, W. Y. Chang, L. C. Tsai, C. H. Chen, and W. H. Yang, 2008: Seismo-ionospheric precursors of the 26 December 2006 M 7.0 Pingtung earthquake doublet. Terr. Atmos. Ocean. Sci., 19, 751-759, doi: 10.3319/TAO.2008.19.6.751(PT)

\section{INTRODUCTION}

Scientists have extensively discussed observations and theories of electromagnetic phenomena associated with seismic activity (Hayakawa and Fujinawa 1994; Hayakawa 1999; Hayakawa 2000; Hayakawa and Molchanov 2002). Pulinets and Boyarchuk (2004) have proposed the penetration of quasi-DC vertical electric fields generated during earthquake propagation to explain long-period anomalous tendencies (over a few hours) of the ionospheric electron density. On the other hand, scientists (Hayakawa and Molchanov 2002) have indicated that seismo-generated atmospheric gravity (or buoyancy) waves (AGW) could trigger short-period fluctuations (from a few seconds to minutes) in ionospheric quantities (such as vertical motion, electron density, electric field, current, etc.) before the actual occurrence of earthquakes. Predictably, after the 21 September $1999 \mathrm{M}_{\mathrm{w}}$ 7.6 Chi-Chi earthquake, ionospheric phenomena associated with seismic activity in Taiwan have been com-

\footnotetext{
* Corresponding author

E-mail:jyliu@jupiter.ss.ncu.edu.tw
}

prehensively studied (Liu et al. 2000, 2001, 2004a, b, 2006; Chen et al. 2004) and to investigate anomalous pre-earthquake signals in the lithosphere, atmosphere, and ionosphere, a project called 'integrated Search for Taiwan Earthquake Precursors' (iSTEP, $i=1,2002 / 4$ - 2006/3; i = 2, 2006/8 2011/7) has been undertaken (Tsai et al. 2004, 2006). iSTEP consists of a main project and five inter-related sub-projects covering seismological variation, gravity and geomagnetic variation, radar interferometry for detection of surface deformation, ionospheric variation or seismo electromagnetic signals (SES), and statistical analysis of earthquake hazards.

Eight networks, the electrode, magnetometer, corona probe, FM tuner, all sky imager, Doppler sounder, ionosonde, and global positioning system (GPS) receiver, have been setup by the iSTEP project and continuously operated to monitor the SES in the lithosphere, atmosphere, and ionosphere in Taiwan. While the ionosonde and GPS receiver networks respectively observe anomalous long-period variations of the ionospheric electron density and total electron content (TEC), the Doppler sounder system monitors short- 
period abnormal fluctuations in the vertical motion of the reflection layer in the ionosphere. Here, it was found that the F2-peak electron density (or the associated plasma frequency foF2) and GPS TEC often decreased anomalously in the afternoon over a period of $1-5$ days before $\mathrm{M} \geqq 5.4$ earthquakes in Taiwan (Liu et al. 2000, 2001, 2004a, b, 2006). The TEC changes further illustrate that the decreased anomalies mainly appear around the fore coming epicenters and the TEC crests of equatorial ionization anomaly (EIA) tend to lessen and move toward the geomagnetic equator (Liu et al. 2001, 2002). Although the long-period ionospheric earthquake precursors of foF 2 and GPS TEC have been frequently observed, no short period ionospheric fluctuations have yet been reported.

In this paper, measurements of a network of groundbased GPS receivers, a digital ionosonde, and a Doppler sounder during the 26 December 2006 Pingtung, Taiwan offshore earthquake are examined (Fig. 1). Not only the temporal and spatial precursors of the long-period foF 2 and GPS TEC anomalies are presented but for the first time, short-period fluctuations of the ionospheric Doppler shift are reported.

\section{INSTRUMENT AND EXPERIMENT}

The GPS is ideal for monitoring a large area with regard to ionospheric electron density. The system consists of more than 24 satellites, distributed in six orbital planes around the globe at an altitude of about $20200 \mathrm{~km}$. Each satellite transmits signals in two frequencies $f_{l}=1575.42 \mathrm{MHz}$ and $f_{2}=$

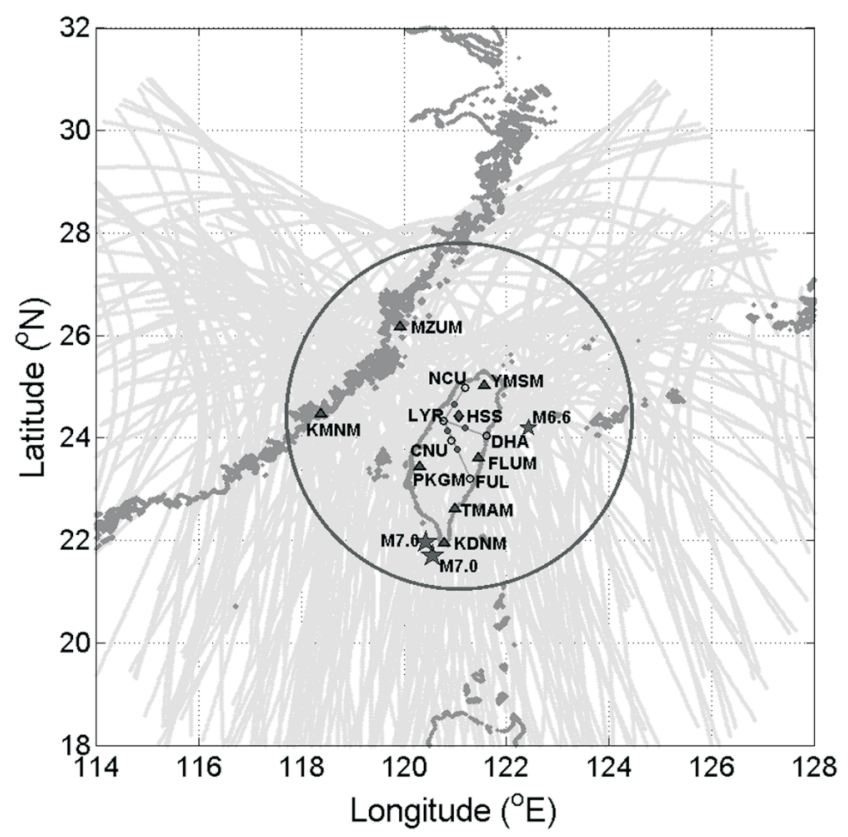

Fig. 1. The location and coverage of the DPS, GPS receiver, and Doppler sounding system. The circle denotes the coverage of the DPS. The gray curves are the GPS satellite ground traces and/or coverage of the receiver network. The detailed locations are given in Table 1.
1227.60 MHz). Since the ionosphere is a dispersive medium, measurements of the modulations on carrier phases and phase codes recorded by dual-frequency receivers can be employed to derive the TEC in the vertical direction, which is written as,

$T E C=\cos \chi \int_{\text {Sat }}^{R x} N d s$

where $N$ is ionospheric electron density, and $\chi$ is the zenith angle of a GPS satellite, sat, at a GPS receiving station $R x$. From the broadcast ephemeris (i.e., the satellite time, elevation or zenith angle, location, etc.) and a given ionospheric (shell) height at $350 \mathrm{~km}$ (Sardón et al. 1994), the associated longitude and latitude (Tsai and Liu 1999) can be found. The TECs together with their associated longitude and latitude with 30 -second time resolution derived by the network are employed to construct an overhead TEC at certain location (such as an epicenter), a daily latitude-time-TEC (LTT) plot, and a snap shot of latitude-longitude-TEC (LLT) map.

The earliest conventional equipment for measuring the virtual height of the ionosphere is a sweep frequency pulsed radar device called an ionosonde. The measurement recorded by an ionosonde is called an ionogram, which displays the variation of the virtual height of reflection with frequency, where the virtual height is equivalent to one-half the timeof-flight of the transmitted radio wave times the speed of light $c$. Due to the Earth's magnetic field, there are two

Table 1. Locations of the GPS receivers, DPS and Doppler Sounding System.

\begin{tabular}{llc}
\hline \multicolumn{1}{c}{ Name } & \multicolumn{1}{c}{ Instrument } & Location \\
\hline HSS & DPS & $24.42^{\circ} \mathrm{N}, 121.08^{\circ} \mathrm{E}$ \\
MZUM & GPS receiver & $26.16^{\circ} \mathrm{N}, 119.93^{\circ} \mathrm{E}$ \\
YMSM & GPS receiver & $25.02^{\circ} \mathrm{N}, 121.57^{\circ} \mathrm{E}$ \\
KMNM & GPS receiver & $24.46^{\circ} \mathrm{N}, 118.39^{\circ} \mathrm{E}$ \\
FLUM & GPS receiver & $23.60^{\circ} \mathrm{N}, 121.45^{\circ} \mathrm{E}$ \\
PKGM & GPS receiver & $23.44^{\circ} \mathrm{N}, 120.31^{\circ} \mathrm{E}$ \\
TMAM & GPS receiver & $22.62^{\circ} \mathrm{N}, 121.01^{\circ} \mathrm{E}$ \\
KDNM & GPS receiver & $21.95^{\circ} \mathrm{N}, 120.78^{\circ} \mathrm{E}$ \\
LYR & DSS* transmitter & $24.34^{\circ} \mathrm{N}, 120.78^{\circ} \mathrm{E}$ \\
NCU & DSS reflection point\# & $24.66^{\circ} \mathrm{N}, 120.98^{\circ} \mathrm{E}$ \\
CNU & DSS reflection point & $24.15^{\circ} \mathrm{N}, 120.85^{\circ} \mathrm{E}$ \\
DHA & DSS reflection point & $24.19^{\circ} \mathrm{N}, 121.19^{\circ} \mathrm{E}$ \\
FUL & DSS reflection point & $23.77^{\circ} \mathrm{N}, 121.04^{\circ} \mathrm{E}$ \\
\hline
\end{tabular}

* DDS: Doppler sounding system.

\# reflection point: the mid-point of the transmitted signal path between the receiver and the transmitter, which is at about $200 \mathrm{~km}$ altitude. 
traces, $\mathrm{O}$-mode and $\mathrm{X}$-mode appearing on the ionogram. Based on the magneto-ionic theory (for detail see Budden 1985), the plasma frequency is equal to the vertically reflected O-mode frequency. The greatest (or critical; penetration) frequency on an $\mathrm{O}$-mode trace, $f_{o F}$, stands for the greatest plasma frequency of the ionosphere. The plasma (or electron) density $N_{p}$ can be derived from the plasma frequency $f_{p}$ and written as (see Davies 1990)

$N_{p}=1.24 \times 10^{4} f_{p}^{2}$

where $N_{p}$ and $f_{p}$ are in el $\mathrm{cm}^{-3}$ and $\mathrm{MHz}$, respectively. From Eq. (2), the ionospheric F2-peak electron density NmF2 can be computed by $f_{p}=f o F 2$. Since the $\mathrm{NmF} 2$ is the greatest electron density in the ionosphere, Eq. (1) indicates that the NmF2 and TEC are highly correlated (also see Liu et al. 2004b).

A high frequency continuous wave (HFCW) Doppler sounding system allows us to monitor the vertical motion at a certain ionospheric reflection point (or layer) of the transmitted signal. The relation between the vertical speed $v$ of the ionospheric layer and the observed Doppler shift $\Delta f$ of the transmitted signal can be written as,

$\Delta f=-\frac{v}{c} 2 f_{R} \cos \theta$

where $f_{R}$ is the sounding radio wave frequency, $c$ the light speed in a vacuum, and $\theta$ is the angle of incident of the sounding wave on the ionosphere. Therefore, variations of $\Delta f$ can be employed to monitor ionospheric vertical motions (fluctuations). The sounding frequency of the system in Taiwan is $5.26 \mathrm{MHz}$ and the distance between the transmitter and each receiver is less than $100 \mathrm{~km}$. If the reflection layer is at about $200 \mathrm{~km}$ altitude, Eq. (3) reveals that the vertical motion $v=1 \mathrm{~m} \mathrm{~s}^{-1}$ results in the Doppler shift $\Delta f=0.034 \mathrm{~Hz}$.

\section{OBSERVATION AND INTERPRETATION}

Two M 7.0 offshore earthquakes $\left(21.69^{\circ} \mathrm{N}, 120.56^{\circ} \mathrm{E}\right.$ and $21.97^{\circ} \mathrm{N}, 120.42^{\circ} \mathrm{E}$ ) near Pingtung county occurred at 20:26:21 and 20:34:51 LT (local time) on 26 December 2006. A network of 8 island-wide scattered GPS receivers and a digital portable sounder (DPS), which is a modern digital ionosonde and termed advanced ionospheric sounders (Hunsucker 1991), are operated to respectively observe the ionospheric TEC and electron density (especially NmF2), while a Doppler sounding system is used to probe the Doppler frequency shift of the transmitted signal monitoring the ionospheric vertical motion. Figure 1 illustrates the location and coverage of the GPS receivers, DPS, and Doppler sounding system. The TEC derived from the 8 GPS network receivers cover an area of $15^{\circ}$ to $30^{\circ} \mathrm{N}$ in latitude and $115^{\circ}$ to $130^{\circ} \mathrm{E}$ in longitude, while the DPS ionogram reveals the vertical electron density distributions up to the F2 peak height with a radius of about $300 \mathrm{~km}$. The 4 receivers of the Doppler sounding system monitor ionospheric vertical motions and their horizontal propagations in Taiwan.

Figure 2 reveals the TEC variations above the epicenters derived by the GPS network in December 2006. It can be seen that decreased long-period anomalies (temporal precursors), which are defined as the observed TECs exceed the associated low bounds (LBs), appear on December 18 (D-8), 19 (D-7), and 22 (D-4), 2006. Since an intense geomagnetic storm occurred during December 14 - 17 (universal time; UT), the D-8, D-7, and D-6 (i.e., December 18, 19, and 20) the decreased anomalies are most likely the storm related. Therefore, we focus on the anomalous D-4 day (22 December 2006) and compare it with an arbitrary normal day (for instance, D-5; 21 December 2006). Since the co-located NmF2 and GPS TEC are highly correlated, we then examine changes of the ionospheric electron density recorded by the DPS during the afternoons of the two days. Figure 3 illustrates that the foF2s at 1400 - 1445 LT (LT = $\mathrm{UT}+8 \mathrm{hr}$ ) on D-4 are significantly less than those on D-5. The foF 2 s at 1430 LT (or 0830 UT) of the two days being about 5.7 and $8.2 \mathrm{MHz}$ shows that the $\mathrm{NmF} 2$ of D-4 anomalously decreases about $48 \%\left(=5.7^{2} / 8.2^{2}\right)$ with respect to that of D-5. To further understand signatures of the spatial precursor, data from the 8 GPS stations are used to construct a sequence of LTT plots to monitor variations of the ionospheric EIA along the Taiwan longitude during December 1 - 31 of 2006. Figure 4 shows the EIA crest anomalously decreasing and moving or southward on D-4. It can be seen that the crest occurrence time on D-4 tends to be ahead of the associated medium but is not statistically significant. Although the anomalous features of the electron density and TEC decreases as well as the southward motion are observed, the forthcoming earthquake has not yet been located. A sequence of 2-hourly LLT maps was constructed with a $0.1^{\circ} \times 0.1^{\circ}$ (or $11 \times 11 \mathrm{~km}$ ) grid resolution in latitude and longitude 1 - 30 days before the Pingtung earthquakes developed to locate the epicenters. Figure 5 displays the afternoon LLT maps of the medium of 1 - 30 days before the earthquakes, on D-4, changes from the medium to D-4, and the extreme minimum of the 30-day pre-earthquake period on 22 December 2006 (D-4). The changes show that the GPS TEC significantly decreases in the southern Taiwan area on D-4. It is interesting to find that the extreme minimum areas appearing on D-4 generally coincide with the epicenters of the forthcoming earthquakes at 1400 and 1600 LT.

Due to frequent typhoons, the receivers at DHA, CNU, and FUL have many data gaps. Therefore, we focus on and carry out a statistical analysis on the data recorded at NCU. Figure 6 illustrates the ionospheric Doppler shifts observed at NCU during December 2007. It can be seen that the Doppler shift fluctuates pronouncedly few days (24 - 26 Decem- 
ber 2006) before the earthquakes. A process is applied to isolate the half bandwidth from each original spectrum and a band pass filter of 2 - 10 minutes is further used to analyze the data time series of the isolated bandwidth over a year from 1 November 2006 to 31 October 2007. It is surprisingly to find that the percentage of quasi 3-minute periodicities in the half bandwidth variations significantly increases $0-2$ days prior to the earthquakes. Similarly, 1 - 6 days before a M 6.6 7 September 2007 northeastern offshore earthquake, similar quasi 3-minute periodicities once again expand.

\section{DISCUSSION AND CONCLUSION}

Results in Figs. 2 and 3 show that the NmF2 (or foF2) and GPS TEC anomalously decrease in the afternoon on day 4 (D-4) before the Pintung earthquakes which are in a good agreement with the previous observations for Taiwan (Liu et al. 2000, 2001, 2004a, b, 2006). Liu et al. (2001) observe the afternoon GPS TECs on day 3 and 4 before the $M_{w} 7.6$ Chi-Chi earthquake decrease dramatically and the associated EIA crests anomaly moves southward. Figure 4 shows that the decrease and motion features once again appear on day 4 (D-4) before the Pintung earthquakes. Liu et al. (2001) also find that the GPS TECs centering around the epicenter significantly decrease in the afternoons of day 3 and 4 before the $M_{w}$ 7.6 Chi-Chi earthquake and suggest that seismo-generated vertical electric fields are important. Liu et al. (2006) statistically demonstrates that the chance of observing the decrease anomaly in foF 2 reduces with the distance to the epicenter. These two studies suggest that seismo-generated electric filed around the epicenter can significantly decrease the electron density or TEC in the afternoons 1 - 5 days before the earthquake. Figure 5 shows that compared to the medium of 1 - 30 days before the Pingtung earthquakes, the afternoon GPS TEC significantly lessens on D-4. The difference between the anomaly and associated medium maps

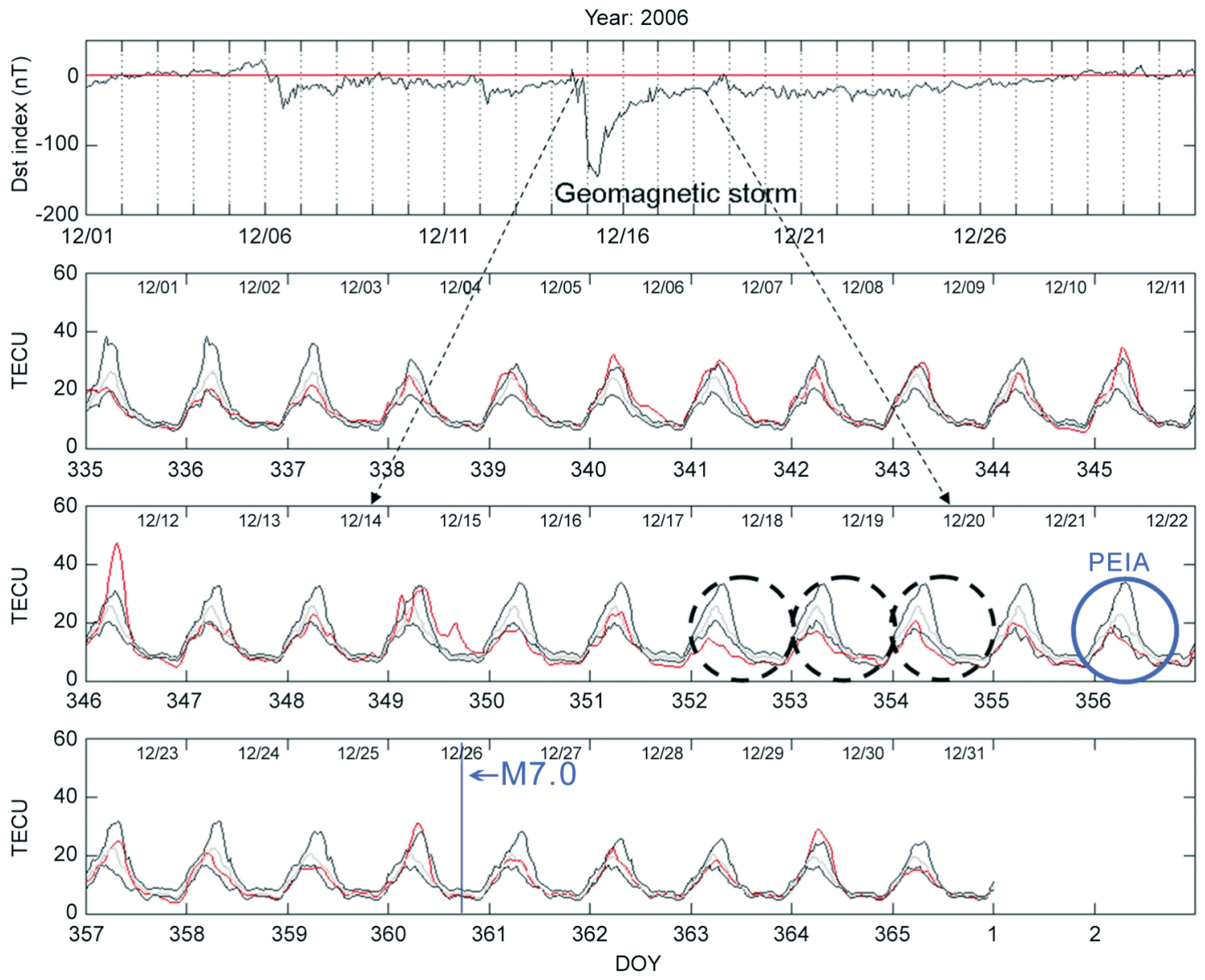

Fig. 2. The Dst index and GPS TEC observed in December 2006. A geomagnetic storm occurred on 14 December 2006. The dashed and solid circles denote the observed TEC exceeding their associated lower bounds. Previous 15-day data are used to construct a reference to identify whether the observation is normal or anomalous. We compute the first (or lower) and the third (or upper) quartiles, denoted by LQ and UQ, respectively. Let M, and $\operatorname{MLR}(=\mathrm{M}-\mathrm{LQ})$ and MUR (= UQ - M) be the median, and the associated lower and upper quartile ranges from the medium, respectively. The lower $\mathrm{LB}(=\mathrm{M}-1.5 \mathrm{MLR})$ and upper bounds UB (= M +1.5MUR) are employed to identify the anomalies. The probability of an observed TEC in the inter$\operatorname{val}(\mathrm{LB}, \mathrm{UB})$ is approximately $75 \%$. 

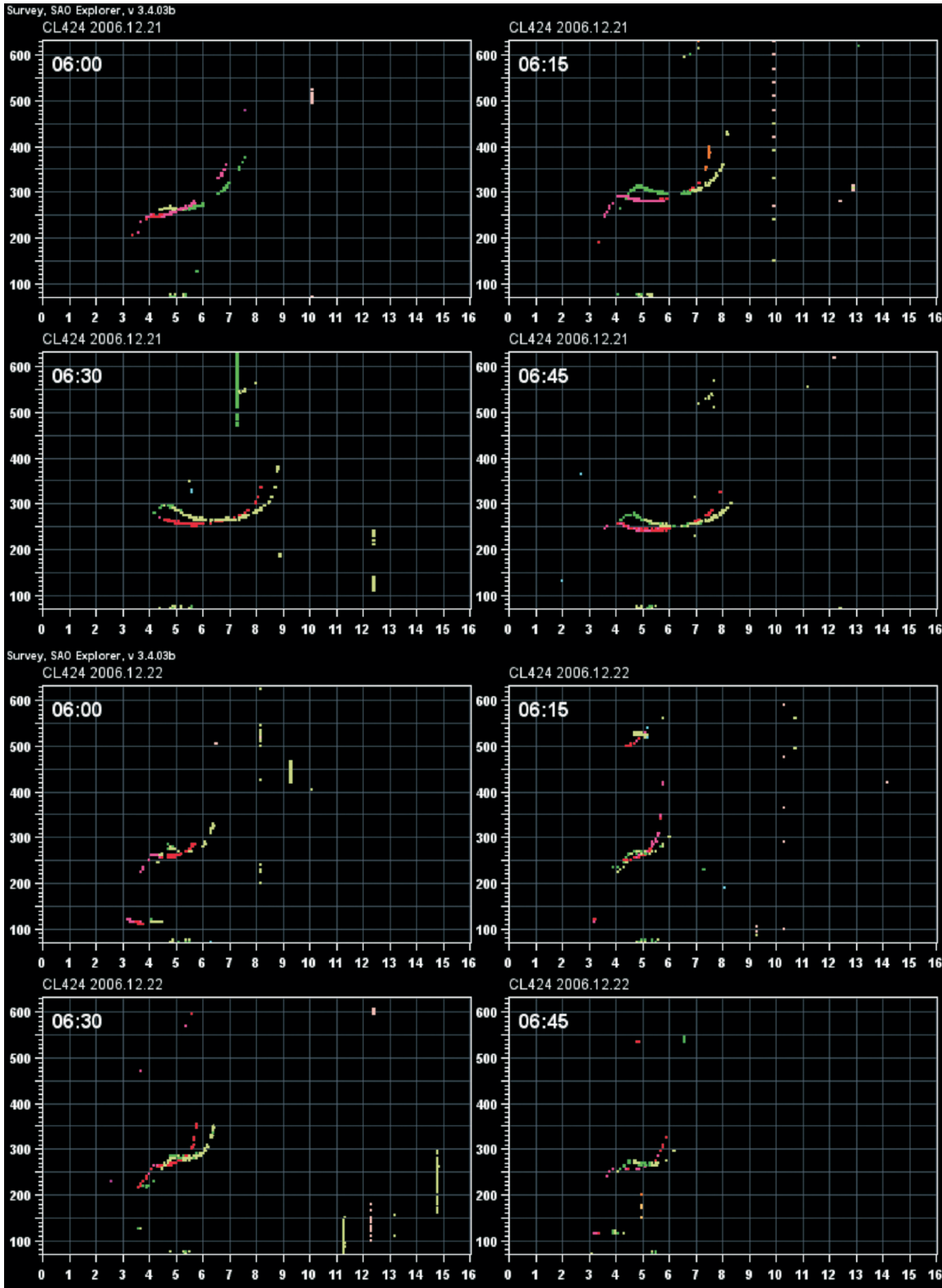

Fig. 3. Ionograms recorded by the DPS during 0600 - 0645 UT on 21 (upper 4 panels) and 22 (lower 4 panels) December 2006. The red and green dots denote the $\mathrm{O}$-mode and $\mathrm{X}$-mode traces, respectively.

confirms that the most significant decease occurs at the epicenters of the Pingtung earthquakes. The 30-day extreme minima further automatically locate the epicenters at 1400 and 1600 LT on D-4.

Based on various emissions, ranging from ULF (ultra low frequency) to VHF (very high frequency), of the electric or magnetic fields in the lithosphere, atmosphere, and lower ionosphere, scientists have predicated that microfractures can somehow trigger AGW near the Earth's surface and in the lower ionosphere (around $70 \mathrm{~km}$ ) during the earthquake preparation period (see papers listed in Hayakawa and $\mathrm{Fu}-$ jinawa 1994; Hayakawa 1999; Hayakawa and Molchanov 
(a)
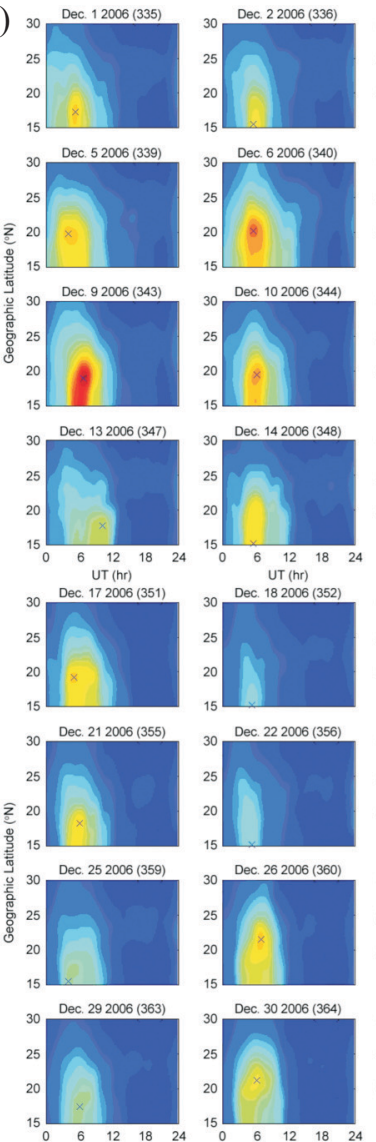

1218

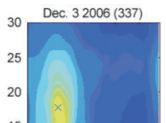

Dec. $72006(34)$
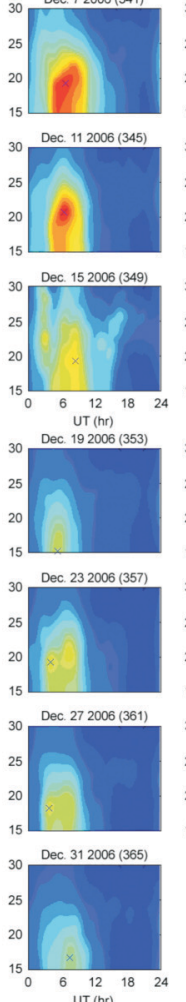

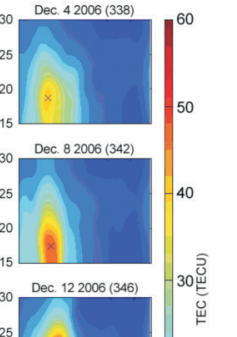

(b)
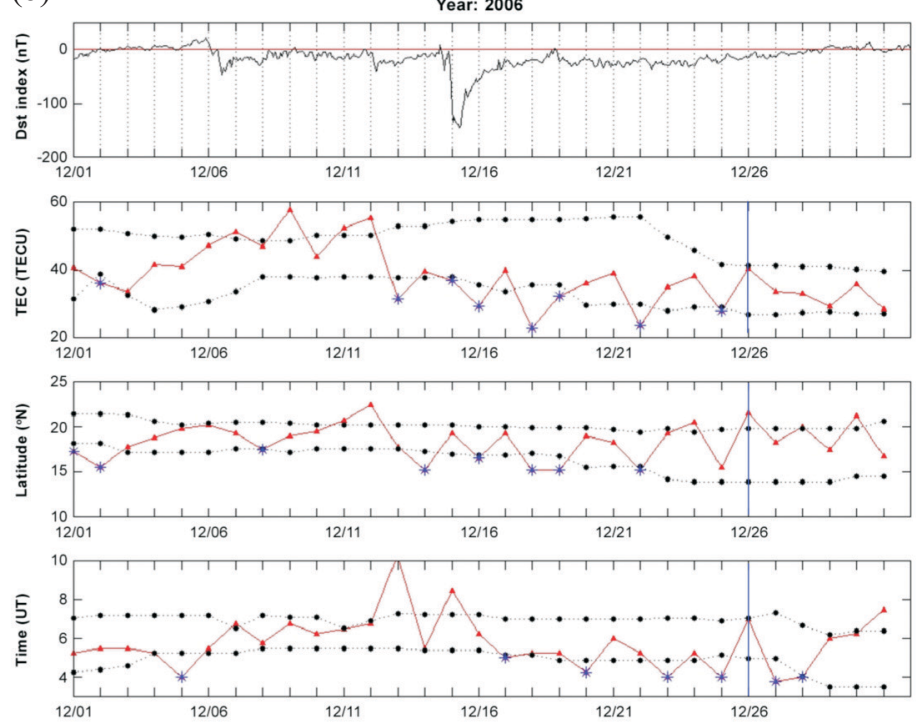

Fig. 4. (a) The LLT maps observed in December 2006. The cross denotes the EIA crest. (b) The Dst, crest TEC, crest latitude, and crest occurrence time extracted from the LLT maps. The solid red and black dotted curves are the observations and associated 15-day running lower and upper bounds. The reference is constructed the same as that found in Fig. 2.
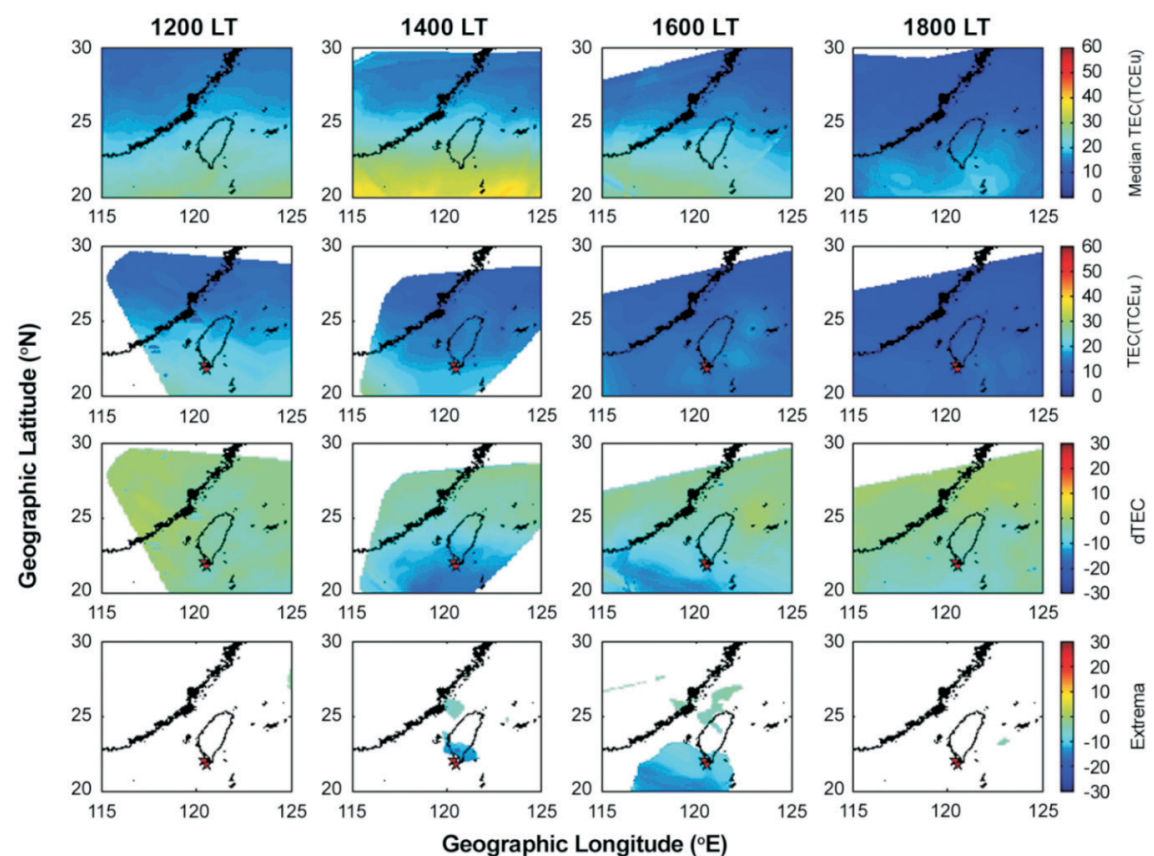

Fig. 5. The LLT maps at 1200, 1400, 1600, and 1800 LT observed prior to the Pingtung earthquakes. The medium of $1-30$ days before the earthquake (top row), observation on D-4 ( $2^{\text {nd }}$ row), the difference of the medium subtracting from D-4 ( ${ }^{\text {rd }}$ row $)$, and the extreme minimum of each grid point in the 30-day period appear on D-4 (bottom panel). The star symbols denote the epicenters of the Pingtung earthquakes. 


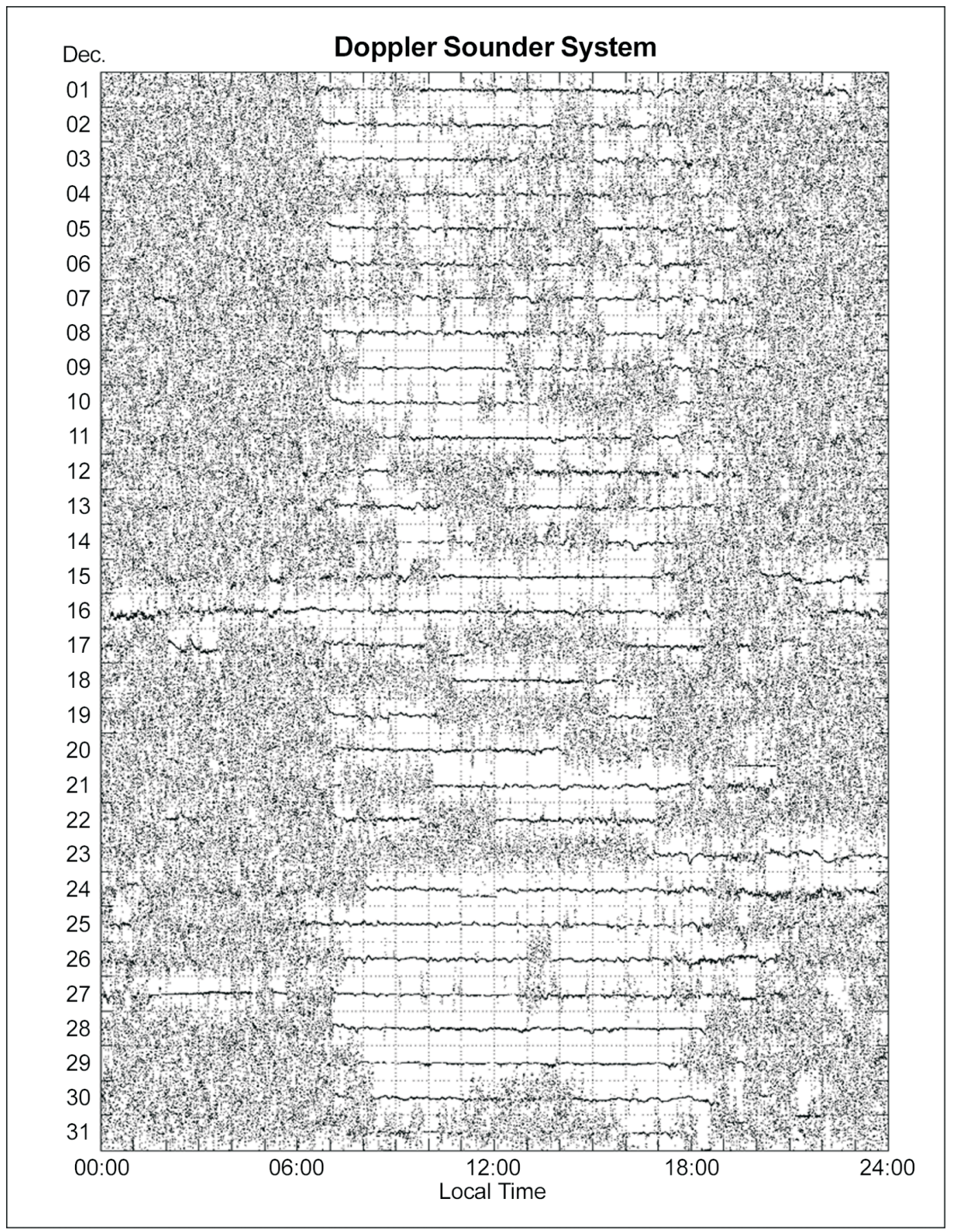

Fig. 6. The Doppler grams observed at NCU in December 2006. The vertical grid denote $\pm 3 \mathrm{~Hz}$.

2002). However, so far, neither the microfractures nor the predicated AGW signature has been yet reported. Figures 6 and 7 display that for the first time the quasi 3-minute AGW features in the ionosphere appear day 0 - 2 before the Pingtung earthquakes. Kunitsyn et al. (2007) and Hsiao et al. (2008) examine tomographic imaging of ionospheric disturbances and wavelike structures and find that seismo-AGWs can be activated near the epicenters which propagate into the ionosphere and then trigger traveling ionospheric distur- bances (TIDs) few days before earthquakes. Coincidently, they also reconstruct ionospheric tomography along the Taiwan longitude in December 2006 and observe seismo-TIDs with $10 \mathrm{~s}-$ minute period and $200 \mathrm{~s}-\mathrm{km}$ wavelength traveling away from the Pingtung epicenters at 0729 LT on D-1 (25 December 2007) and at 0832 LT on D day (26 December 2007). These simultaneous AGWs and TIDs features indicate that the ionosphere becomes highly agitated $0-2$ days before the Pingtung earthquakes. 


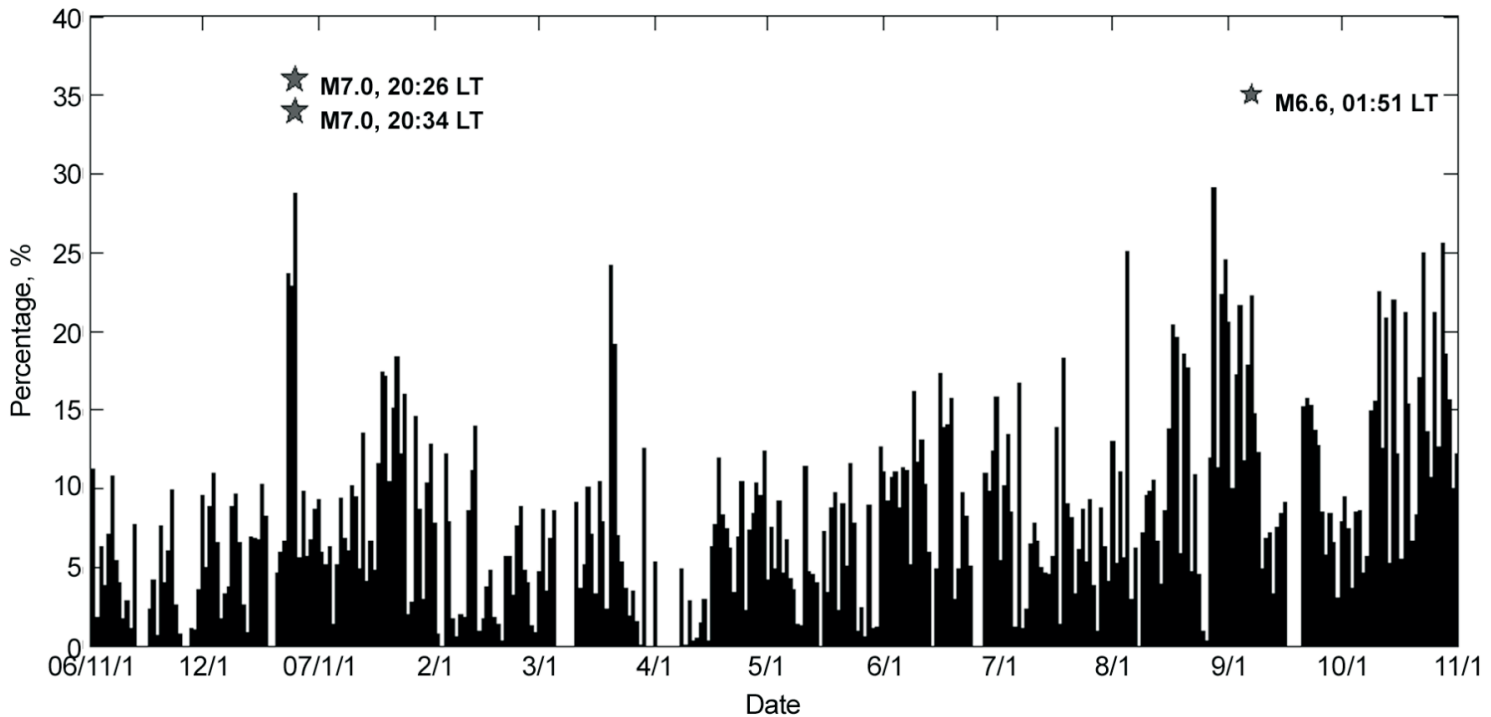

Fig. 7. The percentage of quasi 3-minute fluctuations in the half-band width of the Doppler spectrum observed from 1 November 2006 to 31 October 2007.

Although possible earthquake precursory signatures of the AGW and TID are observed, still no seismic activities associated with the microfractures are recorded. To resolve this discrepancy, we propose a current image model based on the lithosphere and ionosphere being two prefect conductors (Fig. 8). Typical conductivity at about $100 \mathrm{~km}$ altitude for the mid-latitude areas, such as Taiwan, daytime ionosphere is about $100-300 \mathrm{~S} \mathrm{~m}^{-1}$ (or mho m${ }^{-1}$ ) (Kelly 1989)

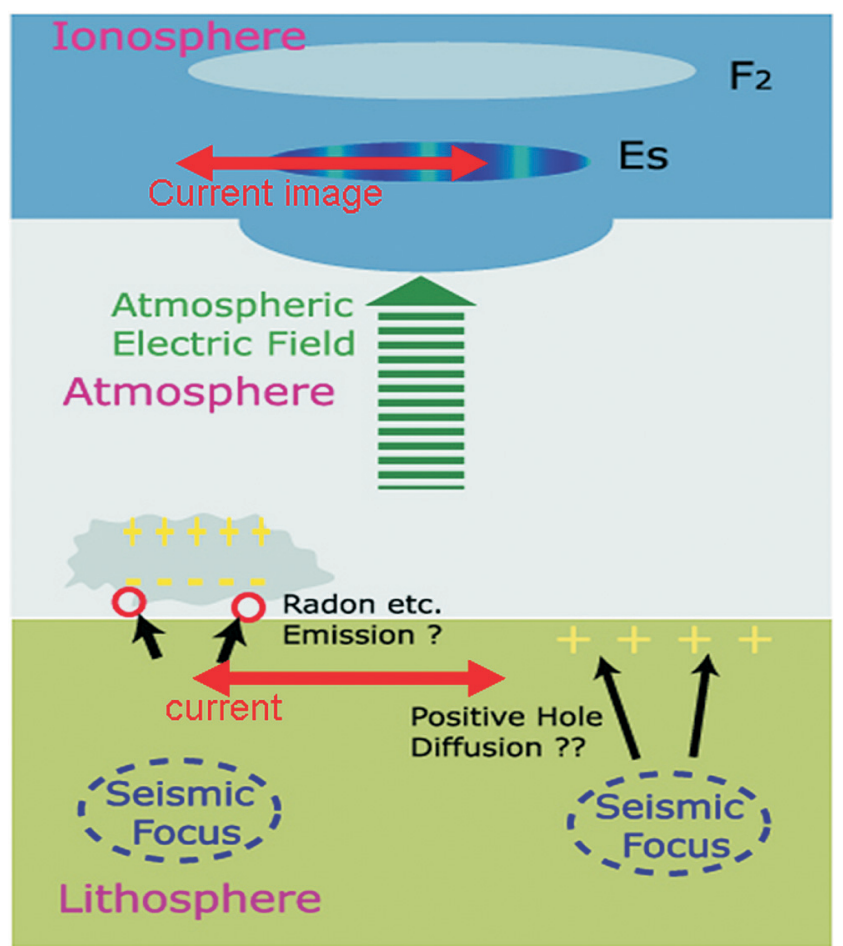

Fig. 8. The seismo-generated current in the lithosphere and its image in the ionosphere. and that for the lithosphere ranges from 0.1 to $1 \mathrm{~S} \mathrm{~m}^{-1}$ at about $40 \mathrm{~km}$ depth (see papers listed in Chen and Chen 1998). Freund (2000) show that mobile positive holes can be activated in the crust by microfractures during the dilatancy stage of earthquake preparation (Bolt 1999), and diffusion and outflow of these holes further generate strong underground currents and high electric fields on the Earth surface. Figure 8 sketches the seismo-generated current in the lithosphere and its image in the ionosphere. Due to Ohm's law, the imaged current dissipates thermal energy resulting in temperature variations and neutral density changes which activate AGUs and TIDs in the ionosphere.

In conclusion, the GPS receiver network and the DPS document the ionospheric electron density (TEC and foF2) around the epicenters which anomalously decrease in the afternoon of Day 4 and the Doppler sounding system detects significantly enhanced quasi 3-mimute AGWs 0 - 2 days before the earthquakes. Both the long and short period earthquake precursors indicate that pronounced SESs have been activated few days before the 26 December 2006 M 7.0 Pingtung earthquakes.

Acknowledgements This research was supported by the Taiwan Earthquake Center (TEC) via the National Science Council project NSC96-2745-M-008-008 as well as Space Science-Technology and Astronomy Center and Earth System Science Center of Top University Plan to the National Central University (NCU). The authors wish to thank Mr. C. H. Chen, Mr. W. H. Yang, Mr. I. T. Lee at the Institute of Space Science of NCU for data analyses and graphing.

\section{REFERENCES}

Bolt, B. A., 1999: Earthquake. $4^{\text {th }}$ Ed., W. H. Freeman, New York. 
Budden, K. G., 1985: The Propagation of Radio Waves. Cambridge University Press, New York.

Chen, C. C. and C. S. Chen, 1998: Preliminary result of magnetotelluric soundings in the fold-thrust belt of Taiwan and possible detection of dehydration. Tectonophysics, 292, 101-117.

Chen, Y. I., J. Y. Liu, Y. B. Tsai, and C. S. Chen, 2004: Statistical tests for pre-earthquake ionospheric anomaly. Terr. Atmos. Ocean. Sci., 15, 385-396.

Davies, K., 1990: Ionospheric Radio. Peter Peregrinus, London, $580 \mathrm{pp}$.

Freund, F., 2000: Time-resolved study of charge generation and propagation in igneous rocks. J. Geophys. Res., 105, 11001-11019.

Hayakawa, M. (Ed.), 1999: Atmospheric and Ionospheric Electromagnetic Phenomena with Earthquakes. TERRAPUB, Tokyo, $997 \mathrm{pp}$.

Hayakawa, M. (Ed.), 2000: Seismo Electromagnetics, Monograph of International Workshop on Seismo Electromagnetics. Tokyo.

Hayakawa, M. and Y. Fujinawa (Eds.), 1994: Electromagnetic Phenomena Relater to Earthquake Predication. TERRAPUB, Tokyo, $667 \mathrm{pp}$.

Hayakawa, M. and O. A. Molchanov (Eds.), 2002: Seismo-Electromagnetics: Lithosphere-Atmosphere-Ionosphere Coupling. TERRAPUB, Tokyo, Japan.

Hsiao, C. C., J. Y. Liu, K. Oyama, N. L. Yen, Y. H. Wang, and J. J. Miaua, 2008: Ionospheric electron density anomaly prior to the 26 December 2006 M7.0 Pingtung Earthquake Doublet observed by FORMOSAT-3/COSMIC. Phys. Chem. Earth, in press.

Hunsucker, R. D., 1991: Radio Techniques for Probing the Terrestrial Ionosphere. Springer-Verlag, Berlin, Heidelberg, New York.

Kelley, M. C., 1989: The Earth's Ionosphere. Elsevier, New York, $487 \mathrm{pp}$.

Kunitsyn, V., E. Tereshchenko, E. Andreeva, M. Nazarenko, I. Nesterov, and A. Padokhin, 2007: Tomographic imaging of ionospheric disturbances and wavelike structures. AOGS $4^{\text {th }}$ Annual Meeting, Bangkok.
Liu, J. Y., Y. I. Chen, S. A. Pulinets, Y. B Tsai, and Y. J. Chuo, 2000: Seismo-ionospheric signatures prior to $\mathrm{M} \geqq 6.0$ Taiwan earthquakes. Geophys. Res. Lett., 27, 3113-3116.

Liu, J. Y., Y. I. Chen, Y. J. Chuo, and H. F. Tsai, 2001: Variations of ionospheric total electron content during the Chi-Chi earthquake. Geophys. Res. Lett., 28, 1383-1386.

Liu, J. Y., Y. J. Chuo, S. A. Pulinets, H. F. Tsai, and X. Zeng, 2002: A study on the TEC perturbations prior to the Rei-Li, Chi-Chi and Chia-Yi earthquakes. In: Hayakawa, M. and O. A. Molchanov (Eds.), Seismo Electromagnetics: Lithosphere-Atmosphere-Ionosphere Coupling, TERRAPUB, Tokyo. 297-301.

Liu, J. Y., Y. J. Chuo, S. J. Shan, Y. B. Tsai, Y. I. Chen, S. A. Pulinets, and S. B. Yu, 2004a: Pre-earthquake ionospheric anomalies registered by continuous GPS TEC measurement. Ann. Geophys., 22, 1585-1593.

Liu, J. Y., Y. I. Chen, H. K. Jhuang, and Y. H. Lin, 2004b: Ionospheric foF2 and TEC anomalous days associated with M $\geqq 5.0$ earthquakes in Taiwan during 1997 - 1999. Terr. Atmos. Ocean. Sci., 15, 371-383.

Liu, J. Y., Y. I. Chen, and Y. J. Chuo, 2006: A statistical investigation of pre-earthquake ionospheric anomaly. J. Geophys. Res., 111, A05304, doi: 10.1029/2005JA011333.

Pulinets, S. and K. Boyarchuk, 2004: Ionospheric Precursors of Earthquakes. Springer-Verlag, Berlin, Heidelberg.

Sardón, E., A. Rius, and N. Zarraoa, 1994: Estimation of the transmitter and receiver differential biases and the ionospheric total electron content from global positioning system observation. Radio Sci., 29, 577.

Tsai, H. F. and J. Y. Liu, 1999: Ionospheric total electron contents response to solar eclipse. J. Geophys. Res., 104, 12657-12668.

Tsai, Y. B., J. Y. Liu, K. F. Ma., H. Y. Yen, K. S. Chen, Y. I. Chen, and C. P. Lee, 2004: Preliminary results of the iSTEP program on integrated search for Taiwan earthquake precursors. Terr. Atmos. Ocean. Sci., 15, 545-562.

Tsai, Y. B., J. Y. Liu, K. F. Ma, Y. H. Yen, K. S. Chen, Y. I. Chen, and C. P. Lee, 2006: Precursory phenomena associated with 1999 Chi-Chi earthquake in Taiwan as identified under the iSTEP program. Phys. Chem. Earth, 31, 365-377. 